\title{
Der Mechanismus des Beckenbodens und das statische Prinzip im Aufbau unsres Körpers.
}

\author{
Von \\ Dr. Max v. Arx, \\ olten (Schweiz). \\ Mit 18 Figuren im Text. \\ Eingegangen am 4. April 1910.
}

Schon vor 13 Jahren hatte ich mich, von rein praktisch-gynäkologischen Standpunkten ausgehend, an das Studium der Beckenentwicklung gemacht, da mich die landläufigen Auffassungen damals schon nicht befriedigten. Die Resultate meiner Arbeit habe ich in drei Fachzeitungen niedergelegt $1,2,3$ ).

Schon damals wies ich auf den engen mechanischen Zusammenhang hin, der zwischen Blase und Uterus einerseits und dem elastischen Beckenboden anderseits besteht. Ich erkannte bereits die Mechanik des Gebärorgans als die eines elastischen einarmigen Hebels usw. und konstruierte bereits, was bisher noch Niemandem gelungen war, das männliche und weibliche Becken des Menschen mit dem Zirkel.

Ich hatte nämlich bei jenen Arbeiten bereits die darstellende Geometrie zu Hilfe genommen, da sie mir eine vorzügliche und zuverlässige Untersuchungsmethode zu sein schien. Ihr hatte ich eine ganze Reihe von Entdeckungen zu verdanken, Entdeckungen, die mich

1) Die Statik der Beckenorgane unter besonderer Berïcksichtigung des Scheidenvorfalls und seiner Beseitigung. Cystocel-Elevation. Korresp. f. Schweiz. Ärzte. 1896. Nr. 13.

2) Geometrie und Statik der weiblichen Beckenorgane. Arch. f. Anatom. u. Physiolog., Anat. Abt. 1896.

3) Über die Ursachen einer natïrlichen Lage des Gebärorgans. VoLkm. Sammlang klinischer Vortrïge. N. F. Nr. 210. 
in hohem Maße befriedigten, wenngleich sie auch noch zusammenhanglos gemacht worden waren.

Die damals gewonnenen Resultate wurden kaum einer Beachtung gewürdigt; nur das praktische Resultat derselben, die Cystocelenoperation, fand da und dort eifrige Anhänger und die Anerkennung, $\mathrm{da} B$ sie auf richtiger Grundlage aufgebaut sei und daher tiberraschend gute Dauerresultate zeitige. Aber immer noch stand man von dem Bann gefesselt da, daß unmöglich die »weichen. Beckeneingeweide einen bestimmenden Einfluß auf die Formgestaltung der Beckenknochen haben könnten.

Erst im Herbst 1908 nahm ich die Studien wieder auf, diesmal mit einem ungeahnten Erfolg. Heute sind wir in der Lage, jedes Beckenskelet nach mathematischen Gesetzen technisch konstruieren zu können. Aber noch weit mehr! -

Mit der Ergründung der Statik des menschlichen Beckens ward uns zugleich auch das physikalisch-mechanische Grundgesetz klar, das dem Aufbau des menschlichen Körpers, wie jedes tierischen Organismus zugrunde liegt.

Wir dürfen uns dieser gewonnenen Resultate freuen; sie werden nicht verfehlen, auf gewissen Gebieten der Medizin und der Naturwissenschaften eine große Vereinfachung in der Denk- und Anschauungsweise herbeizuführen - von ihrem praktischen Nutzen nicht zu reden. Sie lassen sich auch auf exaktem mathematischem und physikalischem Boden leicht diskutieren. An Stelle der Annahme tritt die Konstruktion, an Stelle der Hypothese aber das Gesetz.

$\mathrm{Zu}$ diesen Erfolgen bin ich auf zweifachem Wege gelangt. Fürs erste habe ich, wie früher bei meinen Beckenstudien, unter Zuhilfenahme mathematischer Aufzeichnungen zugleich stets auch die Statik und Mechanik der weichen Bauchorgane berücksichtigt und damit der Forderung Goethes (s. Osteologie, Vortrag II) »nicht nur auf das Nebeneinandersein der Teile zu sehen, sondern auch auf ihren lebendigen, wechselseitigen Einfluß, auf ihre Abhängigkeit und Wirkung", möglichst Genüge getan. Dann bin ich in meiner Untersuchungsmethode noch weiter gegangen und habe diesen ursächlich wechselseitigen Einfluß räumlich nebeneinander liegender Organe auch in zeitlich aufeinander folgenden Stadien der Entwicklung zu verfolgen gesucht, indem ich auch die vergleichende Anatomie und Entwicklungsgeschichte in den Rahmen meiner Untersuchungen einbezog.

"A uf neuen Pfaden der Beckenforschung* habe ich diese Untersuchungen betitelt, deren Resultate in einer Reihe von kurzen 
Vorträgen und Demonstrationen am XIII. Gynäkologen-Kongreß in Straßburg vom Juni 1909 und bald darauf am XVI. Internationalen KongreB in Budapest mitgeteilt wurden. Der Zweck dieser Zeilen ist, eine Übersicht und Orientierung über die Methodik und die gewonnenen Resultate zu geben.

Zunächst wurden ein männliches und ein weibliches Beckenskelet ron normaler Beschaffenheit in der s. Z. von Herm. Mexer als statisch richtig beschriebenen Weise an die Pubo-spinal-Ebene gestellt, d. b.

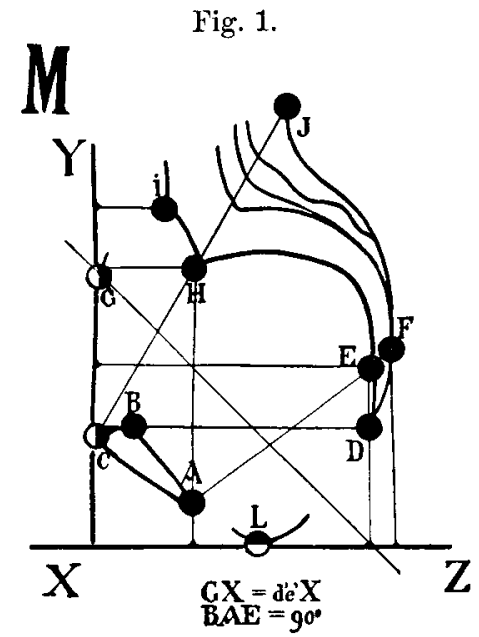

Fig. 2 .

Fig. 1. Projektion der Medianebene eines normalen männlichen Beckens.

$A$ Scheitel des Angulus pubis, $B C$ oberer Rand der Symphyse, $D$ Spitze des Steißbeins, $E F$ unterste Punkte des Kreuzbeins, $G$ Verbindungslinie der Spin. ant. sup. oss. ilei, $H$ Promontorium, $I$ oberer Rand des V. Lendenwirbels, $J$ Proc. spinos. des $V$. Lendenwirbels, $L$ Tuber ischiadicum, $X \mathrm{r}$ Vertikalebene (Abszisse), $X Z$ Horizontalebene (Ordinate).

Fig. 2. Projektion der Medianebene eines normalen weiblichen Beckens.

$A A^{\prime}$ unterster Rand der Symphyse am Arcus pub., $B$ Ausgangspunkt der Conjugata vera, $C$ Tubercala pubis, $D$ Steißbeinspitze, $E F^{\prime}$ unterste Punkte des Kreuzbeins in der Symphysis sacro-coccygea, $G$ Verbindungslinie der Spin. ant. super. oss. ilei, $H$ Promontorium, $I$ oberer Rand des V. Lendenwirbels, $J$ Proc. spinosus des V. Lendenwirbels, $L$ Tuber isehiadicum, $X Y$ Vertikalebene, $X Z$ Horizontaleben $\theta$.

so, daß vorn die beiden vorspringenden Punkte der Schambeinäste und zugleich die beiden Spinae anter. sup. ossis ilei in die Vertikalebene zu liegen kommen. (s. Fig. 1 und $2 C$ und $G$ in $X Y$ ), während das Becken mit seinen Tubera ischii $L$ auf der Horizontalen $X Z$ aufruht. Dabei ist nun von vornherein schon auffallend, daß beim männlichen Becken sowohl wie beim weiblichen 6 Punkte, nämlich 2 Spinae anter. sup., 2 Tubera pubis und 2 Tubera ischii in zwei Ebenen liegen, die in einem rechten Winkel zueinander stehen. In der Horizontalprojektion fallen selbstverständlich die zwei analogen Punkte $G G$, $C C$ and $L L$ je in einen Punkt $G, C$ und $L$ zusammen. Daran reiht 
sich nun in Fig. 1 und 2 die Projektion der Medianebene in der Weise, da $\beta$ die Lage einzelner in die Augen springender Hauptpunkte des knöchernen Skelets genau auf der Abszisse $X Z$ und Ordinate $X Y$ fixiert wird.

So erhält man eine Anzahl mathematisch berechneter Fixpunkte, die sich gruppenweise zusammenstellen, wie folgt:

$$
\begin{array}{ll}
A\left(A^{\prime}\right) B C & \text { Symphysengruppe, } \\
D E F & \text { Steißbeingruppe, } \\
G H I J & \text { Lendengruppe. }
\end{array}
$$

Die Lage dieser Punkte ist nun beim männlichen und beim weiblichen Becken eine wesentlich verschiedene: Sie verschieben sich sowohl innerhalb der Gruppe selber, als auch die Gruppen als solche unter sich. Aber gerade dieser Lagewechsel der Fixpunkte ermöglicht es uns, gleich dem Astronomen, die Gesetze ihrer Verschiebungen zu eruieren und zu berechnen.

$\mathrm{Da}$ wir nun annehmen müssen, daß jedes Becken wie die ganze Körperform das Produkt seiner eignen Statik und Mechanik sei, so mïssen wir auch an jedem einzelnen Beckenskelet die Formeln seines Entwicklungsgesetzes ablesen können. Sind dieselben aber einmal gefunden, so sollen sie, falls sie wirklich mechanisch-physikalisch richtig sind, sich auch verallgemeinern lassen.

Nach dieser Deduktion werden also Messungen zur Ergründung der Statik, an einer endlosen Serienreihe von Beckenskeleten vorgenommen, überflüssig werden. Wir stutzen uns zukünftig auf die mathematische Konstruktion. -

Wir bestimmten nun die Lage der einzelnen Punkte durch Projektion auf Abszisse und Ordinate durch folgende Abstände vom Nullpunkt $X$ (s. Fig. 1 und 2)

am männlichen

Abszisse $X Z$

$$
\begin{array}{cccc}
A & a^{\prime} & 4,75 & \mathrm{~cm} \\
L & & 7,9 & - \\
D & d^{\prime} & 13,5 & - \\
E & \boldsymbol{e}^{\prime} & \mathbf{1 3 , 5} & - \\
F & f^{\prime} & \mathbf{1 4 , 2 5} & - \\
H & h^{\prime} & 4,75 & - \\
\text { dinate } & X Y & \\
A & a^{\prime \prime} & 2,0 & \mathrm{~cm} \\
B & b^{\prime \prime} & 5,6 & -
\end{array}
$$$$
\text { Ordinate } X Y
$$

am weiblichen Becken

$$
\begin{array}{llll}
A & a^{\prime} & 4,0 & \mathrm{~cm} \\
L & & 8,0 & - \\
D & d^{\prime} & \mathbf{1 2 , 5} & - \\
E & \boldsymbol{e}^{\prime} & \mathbf{1 3 , 7 5} & - \\
F & f^{\prime} & 15,0 & - \\
H & h^{\prime} & 5,9 & - \\
& & & \\
A & a^{\prime \prime} & 2,5 & \mathrm{~cm} \\
B & b^{\prime \prime} & 4,5 & -
\end{array}
$$




$\begin{array}{lrrrrrrrr}C & & 5,4 & \mathrm{~cm} & C & & 3,75 & \mathbf{c m} \\ D & d^{\prime \prime} & 5,6 & - & D & d^{\prime \prime} & 6,6 & - \\ E & e^{\prime \prime} & 9,0 & - & E & e^{\prime \prime} & 8,7 & - \\ G & \mathbf{1 3 , 5} & - & G & \mathbf{1 3 , 7 5} & - \\ H & h^{\prime \prime} & 13,9 & - & H & h^{\prime \prime} & \mathbf{1 4 , 2} & - \\ I & i^{\prime \prime} & 17,0 & - & I & i^{\prime \prime} & 17,6 & -\end{array}$

Bevor wir diese Zahlen in ihrem gegenseitigen geometrischen Verhalten näher würdigen, möchten wir nur noch kurz auf einen Punkt aufmerksam machen: Sollte es bisherwirklich noch keinem Forscher aufgefallen sein, daB die hintere Kontur des Kreuzbeins in der Seitenansicht einem reinen Kreissegment entspricht, als dessen Fortsetang schon makroskopisch - beim Manne wenigstens - die vordere Kontur der Symphyse zu betrachten ist? - In der Literatur finde ich dieser Tatsache nirgends Erwähnung getan.

Aus den Zahlen aber, wie sie uns die Projektion der Medianebenen der beiden Becken geliefert, gehen ohne weiteres folgende wichtige Sätze hervor, die wir am besten durch Vergleich der Fig. 1 und 2 uns einprägen können:

1) Beim männlichen Becken geht die Verbindungslinie der Steißbeinspitze $D$ mit dem Angulus pubis $A$ in ihrer Verlängerung nach vorn durch den Nullpunkt $X$ des Koordinatensystems.

2) Beim weiblichen Becken dagegen geht diese statisch wichtige Linie vom Nullpunkt durch den Schnitt des Arcus pubis zum untersten Punkt des Kreuzbeins $E$ in der Symphysis sacro-coccygea (»Projektion der Beckenbodenebene ).

3) Beim Manne liegt das Promontorium $H$ über dem Angulus pubis, die Steißbeinspitze auf der Höhe der Schambeinfuge. Daraus ergibt sich rechtwinklige Verschränkung des männlichen Beckens. Beckenbodenebene and hintere Wand der Symphyse stehen ebenfalls im rechten Winkel zueinander (s. Fig. 16).

4) Beim Weibe kommt das Lot des Promontoriums hinter den Arcus pubis zu liegen; die Steißbeinspitze liegt höher als die Schambeinfuge. Das Steißbein ist infolge der stärkeren Spannung des Beckens nach vorn angezogen und daher weit beweglicher geworden als beim Manne. Mechanisch gehört es nicht mehr der stabilen hinteren Beckenwand an, sondern es bildet beim Weibe eine Vermittlungsrolle als zweite bewegliche, elastische Feder im Beckenboden.

5) Damit ist dokumentiert, daß vom Standpunkt der Statik und Mechanik aus als unterster Punkt der Wirbelsäule aufzufassen sind: 
a) beim Manne die unterste Steißbeinspitze $(D)$,

b) beim Weibe der unterste Kreuzbeinpunkt $(E)$;

d. h. das Steißbein ist beim weiblichen Becken aus der Statik ausgeschaltet, erhält dafür aber eine erhöhte mechanische Bedeutung.

6) Auch der Basiswinkel wollen wir hier im Zusammenhang nochmals gedenken. Sie sind die beiden Winkel eines gleichschenkligen Dreiecks, auf dessen Spitze $A$ die Symphysis pubis aufrubt (Fig. 1 und $2 ; A=$ Angulus, bzw. Arcus pubis). Der »Öffnungswinkel « des Beckens ist gleich dem Spitzenwinkel des Dreiecks.

7) Dieser Öffnungswinkel beträgt beim männlichen Becken

$105^{\circ}$, die Basiswinkel je $371 / 2^{\circ}$

$$
2 \times 371 /^{\circ}=180^{\circ}-105^{\circ} \text {; }
$$

beim weiblichen Becken dagegen ist er

$$
\begin{gathered}
116^{\circ} \text { bis } 120^{\circ}, \text { die Basiswinkel } 30^{\circ} \text { bis } 32^{\circ} \\
2 \times 32^{\circ}=180^{\circ}-116^{\circ} .
\end{gathered}
$$

Schon diese wenigen Sätze eröffnen uns die weitgehendste Perspektive fur die Erkenntnis der Statik und der Entwicklung des menschlichen Beckens und bringen uns auf Ideen, von denen man sagen muß, daß sie unsre bisherigen Anschanungen uber das physische Wesen unsres Körpers als naiv und laienhaft hinter sich lassen. Sie aber weiter zu verfolgen, würde uns hier zu weit führen. Nur noch eins! -

Eine überraschende Tatsache, wohl die wichtigste von allen, blieb von mir lange Zeit unbeachtet.

Nachdem ich die Entfernungen der Punkte auf Abszisse und Ordinate in genannter Weise fixiert hatte, wurde ich mit einem Male gewahr, daß sowohl beim männlichen wie beim weiblichen Becken, die doch sonst so verschieden sind, je zwei Punkte gleichen Abstand haben, nämlich der Projektionspunkt der beiden Spin. ant. super. $G^{*}$ und der Fußpunkt $e^{\prime}$ des Lotes, das vom Tiefstpunkt des Kreuzbeins $E$ auf die Abszisse fällt. (Beim männlichen Becken fällt dieses Lot durch die Steißbeinspitze D.)

Die Entfernung dieser Koordinatenpunkte vom Nullpunkt $\mathrm{X}$ beträgt zwischen 13,5 (männlich) und 13,75 (weiblich).

Diese Entdeckung ward für mich von eminenter Bedeutung, indem sie meine Ahnung zur Gewißheit erhob. Verbinde ich nämlich diese beiden Punkte durch eine Linie, so steht sie als Hypotenuse eines rechtwinkligen Koordinatendreiecks unter einem Neigungswinkel von $45^{\circ}$. Sollte das beim männlichen wie beim weiblichen Becken 
bloßer Zufall sein? Statisch-mathematische Gesetze kennen keineu Zufall! - Um aber diese Gesetze ableiten zu können, begeben wir uns zu einer kurzen Exkursion in das Gebiet der vergleichenden Anatomie und Entwicklungsgeschichte.

Als Analogon zu der bereits erwähnten mathematischen Projektion am ausgewachsenen Becken, hatte ich in Straßburg an der Hand von Präparaten und Medianschnitten menschlicher Friichte und von Bildern aus Werken hervorragender Autoren zuerst darauf aufmerksam gemacht, daß die menschliche Frucht zur Zeit ihrer intrauterinen Entwicklung, und selbst noch bei der Geburt, topographisch-

Fig. 4.

Fig. 3.
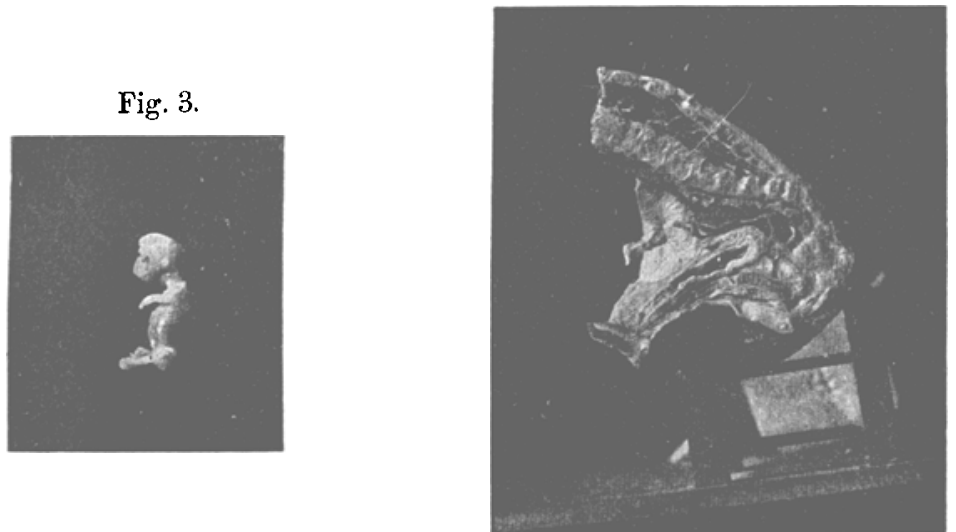

Fig. 3. Henschlicher Fötus aus der 5 . Woche, $3.6 \mathrm{~cm}$ KopfsteiBlānge. Die Masse der festen Brust- und Baucheingeweide formt Ruckgrat, Brustkorb und Lendenschaufeln. Siehe Bum, Grundriß d. Geburtshilfe. Bergmann. 19เ2. Fig. $\$ 1 \mathrm{f}, \mathrm{S} . \$ 3$.

Fig. t. Der Urachusschlauch mit Uterus bei einer 5 Aonate alten menschlichen Frucht, oberhalb der langgestreckten Symphyse in der Bauchhōhle liegend. Wirbelsāule leicht längsgedrückt und zweimal abgebogen.

anatomisch durchaus ähnliche Verhältnisse aufweist wie der Quadruped in bezug auf die örtliche Lagerung der Bauch- und Beckeneingeweide. Der Beckenkanal des neugeborenen Kindes, noch ohne Ausweitung, enthält nur die Ausführungsgänge Urethra, Vagina und Rectum in lockeres Bindegewebe eingehuillt. Blase und Uterus, letzterer als kleiner Appendix der erstern, liegen noch vollkommen in der Bauchböhle. Das hintere Leibesende ist noch spitz und ohne Rundung. - Ich habe weiter gezeigt, daß sich diese ursprünglichen Verhältnisse in der Folge beim ansgewachsenen Tier nur unwesentlich verändern: Blase und Uterus bleiben zeitlebens in der Bauchhöle, das tierische Becken bleibt langgestreckt in liegender Stellung (Fig. 7). - Beim Menschen aber 
wird die Statik bekanntlich später vollkommen umgestaltet. Woran mag das liegen? -

Beim Säugetier und Menschen hat man sich bei der Geburt noch die Urinblase als ungefüllten, elastischen Urachusschlauch zu denken, wie er beim Menschen auch im fünften Fötalmonat noch zu konstatieren ist (s. Fig. 5). Er reicht mit seinem Lumen vom Nabel bis zur Urethralmündung am hinteren Leibesende. Dieser Schlauch füllt sich sodann - sagen wir: nach der Geburt - mit tropfbar beweglicher Fliussigkeit. Zur Erleichterung des Verständnisses setzen wir an Stelle des Urins eine Flüssigkeit von höherem spezifischen Gewicht, z. B. metallisches Quecksilber. Wo wird sich diese Flüssigkeit

Fig. 5.

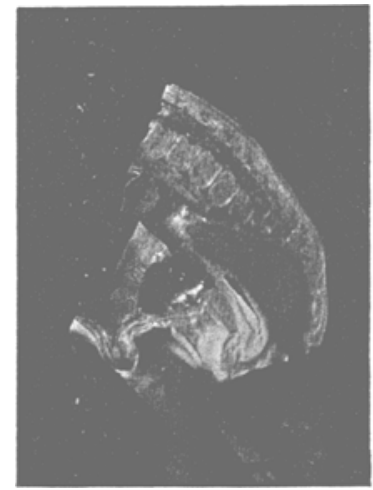

Fig. 6.

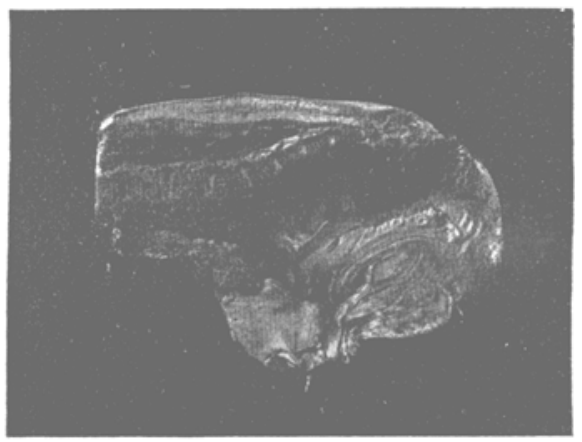

Fig. 5. Beckenmedianschnitt einer 6 Monate alten menschlichen Frucht. Kopfsteißlänge $18 \mathrm{~cm}$. Rectum und Blase injiziert.

Fig. 6. Medianschnitt eines neugeborenen Kindes. Kopfsteißlänge $31 \mathrm{~cm}$. Uterns und Blase befinden sich noch in der Bauchhöhle.

ansammeln? - Doch wohl nur im tiefst gelegenen Punkte dieses Schlauches. Die höher gelegenen Wände desselben sind durch ihre Elastizität und durch die lockeren bindegewebigen Verwachsungen mit der Umgebung in den Stand gesetzt, nachzugeben und diejenige Form anzunehmen, welche für die Statik des flussigen Inhaltes die günstigste ist.

Beim Quadrupeden, der sich gleich nach seiner Geburt auf den Banch legt, bzw. auf die vier Füße stellt, liegt der tiefste Punkt dieses Urachusschlauches an dessen oberem Ende, gegen den Nabel zu. Hier bildet sich also beim Tier die Blase ans; und auf daß hier die Bauchdecken durch diesen Druck nicht zu sehr belastet werden, stïlpt sich die ganze Bauchböhle tiefer in den Brustkorb hinein, d. h. die Baucheingeweide werden möglichst horizontal zwischen Brust- 
korb und Beckenring ausgespannt und von diesen beiden getragen. Dies müßte nun notgedrungen auch beim Menschen der Fall sein, wenn wir das neugeborene Kind beständig auf dem Bauche liegen lassen würden.

Die sorgende Mutter aber, oder die Pflegerin legen den holden Sprößling des Menschengeschlechtes nicht auf den Bauch, sondern auf den Rticken. Dabei kommt der tiefste Punkt des Urachusschlauches unterhalb der Symphyse in das Lumen des Beckenringes zu liegen, und diese Partie wird zur Blase, während ihr oberer Teil gegen den Nabel hin zum Ligamentum medium vesicae obliteriert. Die passive Tragart des Kindes auf dem Arm oder auf dem Rücken der Mutter begünstigt später noch das Tiefertreten der

Fig. 7.

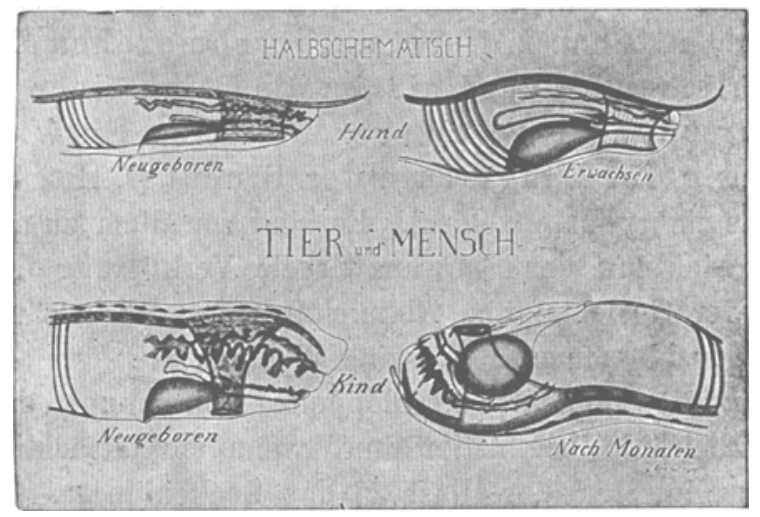

Die Ausbildung der Statik beim Vierfüßler und Menschen nach der Geburt (halbschematisch). Senkung der Blase nach dem kleinen Becken hin infolge der Rückenlagerung des Kindes.

Blase und so kommen jene topographischen Bilder zustande, wie sie uns in schönster Weise Srmivgron in seinem Kinderatlas vorführt: die Blase senkt sich in den ersten Kinderjahren immer tiefer in das kleine Becken hinab. Nar die Schwerkraft und leichte Beweglichkeit des tropfbar flüssigen Blasenmediums (mem. Quecksilber!) hat diese Veränderungen hervorgerufen.

Noch mitssen wir, bevor wir in unsern Betrachtungen weitergehen, auf einen Punkt zurìckkommen. - Der Einwand wird mir nicht erspart bleiben, daß die menschliche Frucht nicht erst bei ibrer Geburt, sondern schon lange vorher, sich ganz wesentlich von der tierischen unterscheide. Ganz gewiß! - Was speziell die Beckenform anbetrifft, hatte ja schon FeHLING vor 30 Jahren an einer großen Reihe von menschlichen Früchten nachgewiesen, daß die Lenden- 
kreuzbeinwirbelsäule rom vierten Monat des intrauterinen Lebens ab eine doppelte Abknickung zeigt. Eine bestimmte Erklärung für diese Tatsache hat Fehling indessen nicht gegeben. Nach Macken. Rodt, Srumpton u. a. fehlen diese Abbiegungen selbst beim Neugeborenen. Das darf uns nicht in Staunen setzen. In utero kom $\mathrm{mt}$ der Frucht durchaus keine eigene Statik zu; die ganze Formgestaltung ist von den inneren und äußeren Druckverhältnissen des mutterlichen Uterus abhängig. Da dieser aber selbst wieder in seiner Form von der Gestalt und dem Mechanismus der ganzen Rumpfhöhle abhängt, erscheint er beim Menschen wesentlich anders als bei den Quadrupeden. Er nimmt schon im Beginn der Schwangerschaft eine rein kuglige Gestalt an, während der befruchtete Tieruterus langgestreckt bleibt. Diese Verbältnisse wirken offenbar schon auf die Formgestaltung der Frucht und unschwer lassen sich alle spezifisch anthropomorphen Merkmale am menschlichen Fötus erklären als Folge einer stärkeren ZusammenstoBung bzw. einer gehemmten Entwicklung in der Richtung der Längsachse der Frucht (s. His, Unsere Körperform, Das embryonale Gehirn). Es sind vor allem zu nennen: Gehemmte Entwicklung der Schwanzwirbel, Verkïrzung des Beckenringes and der Gesichtsform, Abknickung der Wirbelsänle vor und hinter dem geschlossenen Beckenring u. a. m.

Das letztere Moment, die doppelte Abknickung der Lendenkreuzbeinwirbelsäule begünstigt nun, wenn sie tatsächlich vorhanden ist, das Eintreten der Blase in das Beckeninnere. Dieser rein physikalische Vorgang hat aber seine großen Konsequenzen. Der Schwerpunkt wird dadurch nach dem hinteren Leibesende hin verlegt. Stärkere Entwicklung von Darmgasen in den vorderen Partien der Bauchhöhle begünstigt diese Verlagerung des Schwerpunktes, und dadurch allein ist die Möglichkeit gegeben, daß das Kind sich mit dem Oberkörper aufrichten kann. So einfach und natürlich diese Verhältnisse sind, bis jetzt sind sie von Anatomen, Physiologen und Anthropologen mißachtet worden. Was man dabei außer Acht ließ zu bedenken, das läßt sich wohl - abgesehen von dem so häufig verwechselten Causalnexus von Ursache und Folge - am besten auf dem Wege eines einfachen Experimentes darstellen.

Nach Kollmane u. a. bildet die Bauch- und Brusthöhle embryologisch ein einheitliches Ganzes; sie bleiben es statisch mechanisch zeitlebens. Wir haben uns nämlich die 
ganze Brust-, Bauch-, Beckenhöhle als eine große elastische Rumpfblase zu denken, deren Wandung ursprïnglich aus derb elastischem Bindegewebe besteht, das phylogenetisch nichts andres darstellt, als zellig geteiltes Protoplasma. Später treten in dieser elastischen Rumpfwand, je nach Bedürfnis, Modifikationen anf. Wo aktive Kontraktion gefordert wird, erscheint Muskelsubstanz, da wo größere Stabilität zur Herstellung einer Gleichgewichtslage erforderlich ist, versteift sich das Gewebe zu Knorpel und später durch Einlagerung von Kalksalzen zum festen Knochen. Daß diese Einlagerung auf gewisse Reize hin erfolgt, ist durch die Untersuchungen von Roux und Jul. WoLfF genugsam erhärtet. Eine andre wichtige, unsre Ansicht stützende Entdeckung wurde auch kürzlich von MEz gemacht, indem er den Unterschied zwischen Pflanze und Tier dahin definiert, daß er sagt: »Das Tier frißt, die Pflanze aber frißt niemals Und den Freßakt definiert MEz dahin: ,Das Tier stülpt sich über die Nahrung*. Unter diesem Stülpakt aber haben wir uns eine Aktivität der elastischen animalen Substanz der Passivität der Nahrung gegenüber zu denken. Sehen wir zu, inwieweit dies auch beim Menschen seine Richtigkeit hat!

Auch hier ist die Ausführung von Mez vollkommen zutreffend, insofern wir unter der *Nahrung « nicht nur das aufgenommene Futter, sondern auch die Atmungsluft verstehen. Außerdem ziehen wir die Secrete und Excrete der Leibeshöhle und die in der Ausstoßung begriffene Frucht heran. Inwieweit wir auch die Blutmenge des Herzens und der großen Gefäße hierin einbeziehen sollen, lassen wir dahingestellt; mechanisch gehört sie ebenfalls zu dieser Gruppe der passiv bewegten Körper.

Alle diese Stoffe sind nämlich der aktiv und passiv elastischen, animalen Substanz gegenüber als relativ tote Körper zu betrachten; wir bezeichnen diese $\gg$ Contenta Materie $\ll$. Als solche unterliegen sie in ihren mechanischen Wirkungen nur den rein physikalischen Gesetzen der Mechanik fester, flìssiger und gasförmiger Körper; die chemischen Vorgänge können wir hier ohne Schaden ignorieren. Eines nur müssen wir stets im Auge behalten: diese tote Materie ist im Körper überall eingeschlossen von elastischem Gewebe (Bronchus, Digestionstractus, Harnleiter und Blase, Uterus). Der Einfachheit halber zerlegen wir auch den Magendarmtractus in eine Anzahl unter sich unabhängiger Darmschlingen, indem wir uns den Darm durch eine Anzahl Ligaturen unterbunden denken. Und nun zu unsrem Experi- 
ment! - Es soll uns die rein physikalischen Vorgänge, die Mechanik und Statik der Rumpfhöhle und ihrer Eingeweide in groben Zügen veranschaulichen.

I. Dazu benutzen wir eine große elastische Blase aus Kautschuk von ovaler Form, in deren Lumen eine zweite kleinere elastische Blase von mehr kugelförmiger Gestalt eingelassen ist, in der Weise, daß sie an einem Punkte an der Innenwand der Mutterblase befestigt ist und hier durch ein kleines Röhrchen gefüllt und entleert werden kann. Die größere Mutterblase kann durch ein andres Röhrchen ebenfalls gefüllt werden. Nachdem diese große, der Rumpfhöhle entsprechende Blase $A$ mäßig mit Luft aufgetrieben worden, fullt man die innere Kugel $B$ (Urinblase) mit Wasser. Dadurch wird auch der Druck in der Mutterblase erhöht und ihre Wandung überall gleichmäßig gedehnt.

II. Wir fullen eine andre gleichgroße Rumpfblase $A$ statt mit Luft mit einer Anzahl kleiner luft- und fluissigkeithaltiger Blasen und wiederholen dasselbe Experiment, indem wir die vorher entleerte Blasenkugel $B$ wieder mit Wasser füllen. Von der Erhöhung des Druckes werden nun nicht nur die Mutterblase, sondern auch die kleineren Blasen $C C$ getroffen. Infolge der Ausdehnung und Gestaltsveränderung der Rumpfblasenwand aber verschieben sich diese kleineren Kugeln lebhaft aneinander; die specifisch leichteren drängen sich dabei nach oben; wo ihr Inhalt schwerer ist, senken sie sich mehr nach unten. Diese Verschiebungsfähigkeit ist abhängig von verschiedenen Faktoren:

a) von dem Elastizitätsvermögen der äußeren Blasenwand,

b) von dem relativen spez. Gewicht der Contenta ) der Tochter-

c) von dem Elastizitätsgrad der Wandung blasen $C C$,

d) ron der Beschaffenheit der Wandungen bzw. dem Reibungskoeffizienten der Tochterblasen unter sich und an der Innenfläche der Rumpfblase,

e) von etwaigen Befestigungsverhältnissen einzelner Blasen unter sich oder mit der Rumpfblasenwand.

III. Wir erböhen die Vergleichbarkeit dieses Experimentes mit dem Lebensgeschehen dadurch, daß wir Experiment I und II miteinander verbinden und von dem einen Ende der ovalen Rumpfblase eine größere Blase oder ein durch Zwischenwandung abgeschlossenes Fach durch das Röhrchen mit Luft auftreiben und in regelmäßigen Intervallen wiederum entleeren (Lungenalveole über dem Zwerchfell). 
Durch diese Experimente haben wir in einfacher Weise die Grundzüge der Physik und Mechanik in unsern Körpereingeweiden manifestiert. Unschwer lassen sich nun durch mathematische Berechnung die genaueren Gesetze der Mechanik ableiten.

Was nun die Statik dieser länglich-ovalen Blase anbetrifft, so kann ihre Stellung und Lage eine verschiedene sein, und zwar:

a) eine rein horizontal liegende (Fig. 8 und 9 ,

b) eine rein vertikal stehende,

c) eine geneigte (Fig. 10).

Fig. 8.

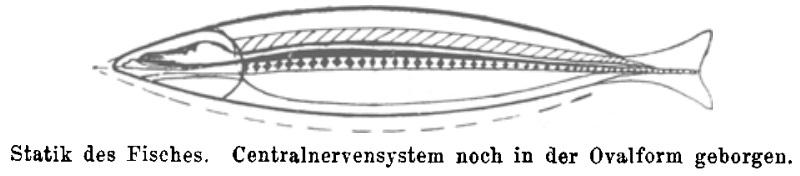

Die horizontale Lage finden wir, wie wir bereits oben dargestellt haben, z. B. beim Fisch und beim Quadrupeden. Da die Versteifung der Rumpfwand hier ebenfalls meist eine horizontale Lage hat, so daß die Rumpfblase an ihr aufgehängt erscheint, ruht sie im stabilen Gleichgewicht; die statischen Verhältnisse sind daher beim Tier sehr

Fig. 9.

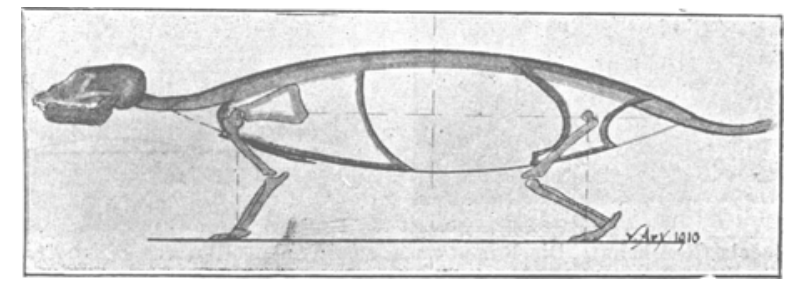

Statik der längs-ovalen liegenden Rumpfblase des Vierfüßlers.

Das Centralnervensystem ist ans der Rumpf blase herausgewachsen. Schematisch.

gute; weniger gut ist die Mechanik, welche die tote Materie der Contenta horizontal weiter za befördern hat.

Die rein vertikal aufrechte Stellung der Rumpfblase wäre bis jetzt immer als eine beim Menschen vorliegende angesehen worden, wenn man überhaupt derselben eine statische Existenz eingeräumt hätte. Denn alle Autoren, ohne Ausnahme, sind bei ihren Untersuchungen am Menschen stets von seiner »aufrechten vertikalen Stellung * ausgegangen. Diese Lage der ovalen Mutterblase wäre wohl für die Mechanik der Eingeweide die denkbar günstigste, indem die Contenta nur der Schwerkraft folgend gegen das hintere, also hier untere 
Leibesende befördert würden. Dadurch aber würde dieser Teil der Rumpfwand ubermäßig belastet werden, so daß entweder eine Ossifikation des ganzen unteren Leibesendes eintreten müßte, oder aber eine vollständige Eventeration der Bauchhöhle. Aus diesen Gründen ist die rein vertikal-aufrechte Stellung des Leibes für den tierischen und menschlichen Organismus eine physikalische Unmöglichkeit.

Nun bleibt für den menschlichen Rumpf nur noch die dritte Möglichkeit der Lage: es ist die geneigte. Diese geneigte Stellung einer walzenförmigen oder ovalen Blase hat aber statisch zwei Varie-

Fig. 10.
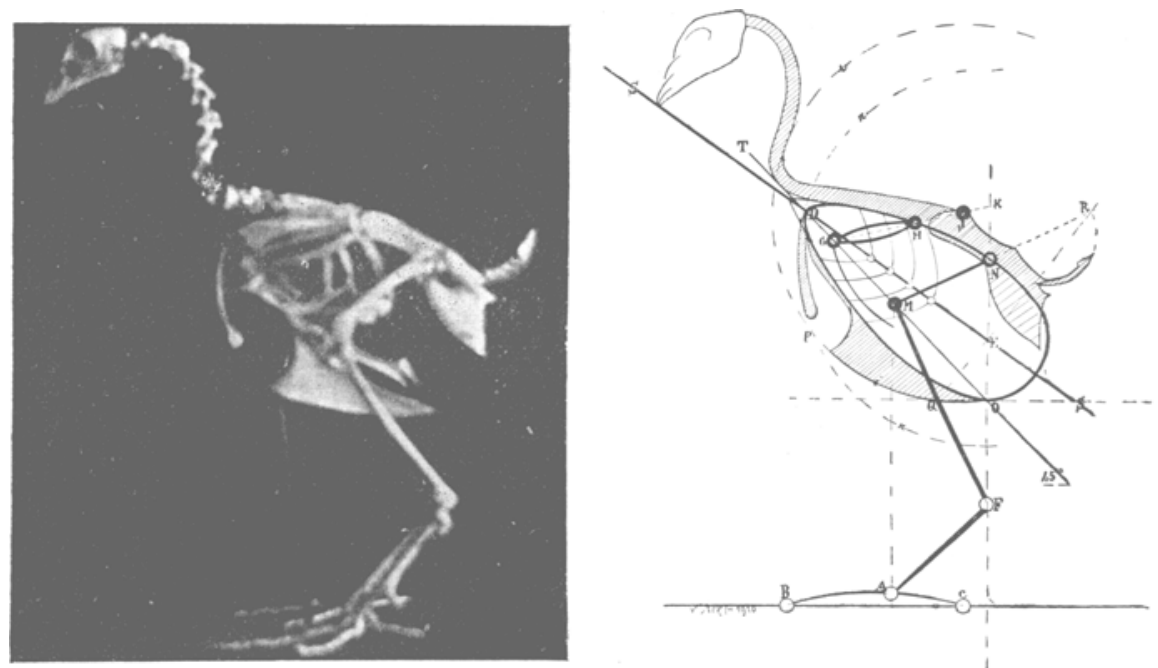

Doppelstatik des Vogels (Hanshahn). Die Rampfblase ist eiförmig. Humerus $I / H$ irrtümlich weggelassen.

täten. Sie kann entweder im stabilen oder im labilen Gleichgewicht sein, was ubrigens auch, nebenbei nachgeholt, auch bei der horizontalen und vertikalen Lage der Fall sein könnte. Diese doppelte Gleichgewichtslage kommt nun auch beim Körperbau des Vogels vor, je nachdem das Tier steht oder sich im Fluge befindet (Fig. 10).

Am allergünstigsten für Mechanik wie Statik wäre nun die Schiefstellung des Rumpfes im labilen Gleichgewicht mit Aufhängepunkt in der Schultergegend; ihr entspricht die Statik des fliegenden Vogels, oder noch besser gesagt, des schwebenden Engels, und es ist höchst amiisant zu sehen, daß diese mythische Figur, die von alters her stets als das ethische Ideal der Menschheit angesehen wurde, zugleich sich auch als ihr physisches Vorbild entpuppt. 
Der Rumpf des Menschen aber steht in Schrägstellung, im stabilen Gleichgewicht mitkonsekativer Abknickung desoberen Rumpfendes zur Vertikalen. Das ist der Fundamentalsatz unsrer Entdeckung. Der physikalische Grund aber, der diese Stellang ermöglicht, ist die Senkung der Blase nach dem kleinen Becken hin.

Kehren wir in unsern Betrachtungen wieder zum Säugling in der Wiege zuriuck! Wir haben ihn aus den Augen gelassen, als ihn die Mutter zum erstenmal aufrecht sitzen ließ".

Nach wenig Monden ist durch die geschilderten Vorgänge die definitive Verlegung des Schwerpunktes so weit gesichert, daß nun auch alle ubrigen Teile des Körpers beginnen, sich der neuen Lage anzuschmiegen. Die Rumpfblase wird wie beim Vogel auf den hinteren Pol gestellt und damit wird das Becken zu ihrer Basis. Darin liegt die hervorragende Bedeutung des Beckenringes für die Statik des menschlichen Körpers.

Man hat bis jetzt wohl auch zuweilen einen vulgären Vergleich gezogen, indem man das Becken mit einer Schüssel verglich, welche die Eingeweide trage. Nichts ist aber falscher, das richtige Verständnis schädigender, als das! Bei einer solchen Schüssel, in welche offen eine Anzahl von Eingeweiden gelegt werden - wir wollen den Vergleich möglichst genau wiedergeben - lastet das ganze Gewicht der letzteren allein auf dem Boden der Schussel, indem sich die Eingeweide möglichst flach darüber hinlegen. Dies ist aber am Becken nicht einmal in der Belastung der Darmbeinschaufeln der Fall, die doch zunächst zum Vergleich herangezogen werden könnten. Das geht schon daraus hervor, daß die Eingeweide in der Bauchböhle nicht frei liegen, sondern aufgehängt sind, indem sie direkte oder indirekte Ansatz- und Aufhängepunkte an Brustkorb, Wirbelsäule oder vorderer Bauchwand erhalten. Wenn dem aber wirklich so ist, so wäre dadurch schon unsre aprioristische Auffassung tiber das Wesen der Rumpfblase gerechtfertigt. Wäre nämlich der Vergleich mit der Schüssel richtig, so müßten bei jeder Lageveränderung des Körpers die Eingeweide sich totaliter verschieben, in Riickenlage des Individuums vor die Wirbelsäule fallen und in Bauchlage nur die Bauchmuskulatur belasten usw. Das ist in der Tat aber nur in sebr beschränktem Grade der Fall. Infolge des ausgeglichenen Spannungswiderstandes zwischen den einzelnen abgeschlossen gedachten elastischen Hohlräumen der Eingeweide (s. o. S. 336 Exper. II $B$ und $C C$ ) in der Rumpfblase verschiebt sich nämlich beispielsweise der Inhalt einer Darmschlinge bei einer Lageveränderung des Körpers nur inner- 
halb dieser Schlinge, und zwar nur dann, wenn ihr Inhalt zu gleicher Zeit verschiedene Aggregatzustände aufweist, z. B. tropfbar flissigen und gasförmigen. Wie wesentlich verschieden sich in dieser mechanischen Beziehung auch der Inhalt unsrer Körpereingeweide und frei in der Bauchböhle liegende Flussigkeit verhält, welch letztere nur von der Spannkraft der Bauchblase umfaßt wird, können wir leicht bei pathologischen Verhältnissen nachweisen, wo nur der freie Ascites jeder Lageveränderung des Körpers folgt.

Da bei all diesen Experimenten - wie ich bereits gezeigt habe der Elastizitätsgrad der umschließenden Gewebe den wesentlichsten Faktor bildet, so ist leicht daraus ersichtlich, da B Experimente an der Leiche nicht verwendbar sind, sobald einmal nur die geringste Leichenstarre eingetreten ist. Aus dem gleichen Grunde sind auch Schnitte an gefrorenen Leichen nur mit großer Vorsicht aufzunehmen. Als Beweis hierfür führe ich an das verschiedene Verhalten der Baucheingeweide bei Eröffnung der Bauchhöhle intra vitam und post mortem. Je größer nämlich die Spannung und Spannkraft der Bauchwand ist, um so rascher und stärker ist auch bei einer Verletzung derselben an ein und derselben Stelle der Prolaps der Eingeweide. Was dabei vorfällt, ist aber nach chirurgischen Erfahrungen nicht immer das zunächst liegende Organ, sondern dasjenige, dem die größte Bewegungsfähigkeit und die größte Beweglichkeit zukommt. Mit dieser Auffassung stimmen sämtliche Vorkommnisse auf physiologischem und pathologischem Gebiete tiberein; sie erleichtert auch die Erklärungen in der Herniologie und Gynäkologie ganz wesentlich und macht die - sich in ihren Resultaten übrigens so sehr widersprechenden - Untersuchungen uber den intraabdominalen Druck gänzlich uberflüssig.

Der Vergleich des Beckens mit der Schüssel hinkt aber hauptsächlich noch aus einem weiteren Grunde. - Das Beckenskelet ist tatsächlich in seinem Formprinzip gar keine Schüssel, sondern ein knöcherner Ring, und zwar ein starr elastischer Ring, der unten durch Muskelgewebe und Fascien von höherer Elastizität geschlossen erscheint. Aus diesem Grunde müssen die mathematischen Punkte des Ringes, auch wenn sie in ihrer gegenseitigen Lage durchaus nicht fix sind, dem beweglicheren, elastischen Beckenboden gegenüber doch als Stütz- und Fixpunkte gelten. DaB die einseitige Belastung des Beckenbodens aber mit der Zeit seine Elastizitätskraft uberwältigen und dadurch zur völligen Eventeration der Bauchhöhle führen müßte, darauf haben wir schon oben hingewiesen. 

Auch wäre die völlige Entleerung der Urinblase in diesem Falle ganz außerordentlich erschwert und nur durch Vornahme einer Lageveränderung des Körpers oder unnötigem Kraftaufwand von seiten der Blasenmuskulatur denkbar, indem der Blasengrund auf dem Beckenboden wesentlich tiefer zu liegen käme, als die Ausflußöffnung im Blasenhals. Solche Verhältnisse finden sich freilich pathologischerweise bei der Cystocele des Weibes und der Prostatahypertrophie beim Manne. Bei diesen pathologischen Zuständen nun werden bekanntlich bereits statische und mechanische Kniffe zur Anwendung gebracht, gleichwie beim Entleerungsakt vieler Tiere, sobald die Blase gänzlich entleert werden soll.

Solchen unnötigen Kraftaufwand zu vermeiden, ist die Natur in gleicher Weise bestrebt, wie sie auch nach möglichster Ökonomie in Anwendung des Materials trachtet. Sie erreicht dies dadurch außerordentlich leicht, daß sich die dehnbare Körpermasse stetsfort in statisches Gleichgewicht setzt, und zwar auf folgende Weise:

Da der tropf bar fliussige Inhalt der Urinblase - abgesehen von den expansiven Gasen - den beweglichsten Teil der toten Contenta ausmacht, so muß in erster Linie diese Blase statisch sichergestellt werden. Nachdem aus oben beschriebenen Gründen die Elevation des Rumpfes möglich geworden, richtet sich diese Rumpfblase mit dem beweglichen toten Inhalt so weit auf, bis zunächst die Urinblase mechanisch zur Ruhe kommt. Sie bewegt sich nämlich theoretisch als eine inkompressible Kugel vom Nabel, dem obersten Punkte des Urachusschlauches weg, auf der durch die Elevation der Rumpfblase entstandenen geneigten Unterlage, den beiden Musculi recti und der inneren Schambeinwand, nach unten gegen den Beckenboden hin. Am Nabel wird sie beim Fall auf der schiefen Ebene immer noch fixiert durch das Lig. medium vesicae. Eine weitere Fixation besteht nur noch im Durchtritt der Harnröhre durch den Beckenboden unter dem Angulus pubis. Alle weiteren Verbindungen sind lockeres Bindegewebe, das sich erst nach erfolgter statischer Ausgleichung, soweit notwendig, befestigt und verstärkt. Je mehr sich die Rumpfblase aufrichtet, um so mehr wird die vordere Bauchwand vom Drucke dieser Blase entlastet und diese Last auf den Beckenboden abgeladen. Die Folgen der einseitigen Belastung des Beckenbodens haben wir bereits kennen gelernt; zu erläutern bleibt nur noch in aller Kürze der Begriff dieses Beckenbodens.

Wie man sich an jeder Leiche nach Entfernung sämtlicher Beckeneingeweide leicht uberzeugen kann, bildet dieses Diaphragma pelvis 
ein wohlgeformtes Kugelsegment, das sich aus folgenden flächenhaften Muskeln und Fascien zusammensetzt:

a) Trigonum uro-genitale,

b) Musc. levator ani (portio pelvica, portio iliaca),

c) Musc. ichio-coccygeus.

Das Diaphragma wird sodann noch verstärkt durch die Schließmuskeln der durchtretenden Kanäle, nämlich den

Musc. constrictor ani,

Musc. constrictor cunni, bzw.

Musc. bulbo-cavernosus (vgl. Bumn, Grundr. d. Geb., S. 160).

Fig. 11.

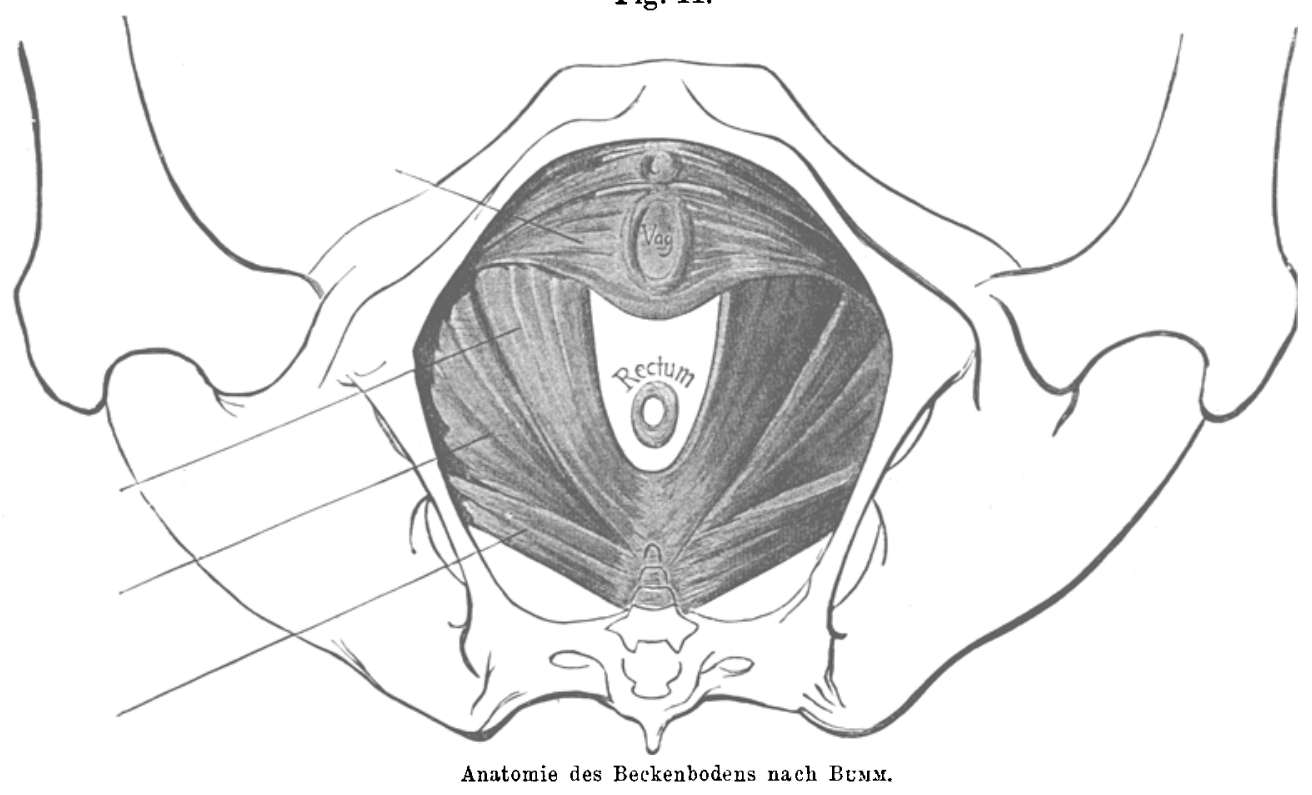

Diese Fascien und Muskeln bilden den eigentlichen Abschluß des Beckens und der Rumpfhöhle nach unten hinten zu. Die knöchernen Beckenteile, welche unter- und außerhalb dieses Beckenbodens liegen, wie die Tubera ischii und der Ramus ascend. ossis pubis kommen für die Mechanik der Becken-Rumpfhöhle nicht mehr in Betracht, sondern nur als direkte oder indirekte Stütz- und Ansatzpunkte für die untere Extremität und ihre Muskulatur.

Für das Kugelsegment des elastischen Beckenbodens aber fällt statisdh in Berechnung die zugehörige Segmentebene, die vorn am Angulus (bzw. Areus pubis) beginnt und sich über die Foramina obturatoria - ligg. spinosa - sacra zum untersten Punkte des Os sacrum 
hinzieht. Diese ideelle Beckenbodenebene wird also durch die untersten Fixpunkte des Beckenringes gelegt. Sie ist im Grunde genommen - beim weiblichen Becken wenigstens - identisch mit der Ebene des geraden Durchmessers des Beckenausganges; beim männlichen Becken, wo das Steißbein weniger beweglich ist, ist dafur die tiefer liegende Ebene zu nehmen, welche hinten durch die Steißbeinspitze geht (Fig. $1 X A D$ ). Auf diese Ebenen nun wird die zweite Hälfte des Gewichtes der Eingeweide abgeladen, bzw. gleichmäßig an den Beckenring aufgehängt.

Das Grundprinzip der Statik und Mechanik des menschlichen Körpers nun erhellt zunächst am besten aus beistehender Fig. 12. $B R$ stellt dabei den Medianschnitt des schematisch und unelastisch gedachten knöchernen Beckenringes dar, der oben und unten durch die elastische Rumpfblase $A E N$ von länglich-ovaler Form geschlossen wird. $A E$ ist Beckenboden, $B N R$ stellt die oberhalb des Beckenringes gelegene Bauch-Brusthöhle dar. Letztere steht unter mäßigem Innendrack gespannt. Im Nabelpunkt $N$ finden wir im Innern dieser elastisch umgrenzten Höhle die inkompressible Harnblase $C$. Die Längsachse $S S$ des Beckenringes und der längs-ovalen Mutterblase ist in den Elevationswinkel von $45^{\circ}$ gestellt.

Wir haben diese Achse $S S$ des Beckenringes mit dem Namen "Seelenachse * belegt; gleichwie in der Ballistik des Geschützrohres, so ist auch in der Beckenstatik dieser Elevationswinkel von $45^{\circ}$ der günstigste von allen.

Die statischen Vorteile dieser Elevationsstellung sind sofort in die Augen springend. Zwar findet von seiten der fallenden Blasenkugel auf ihre Unterlagen (Schambein bzw. Beckenring und Beckenboden bzw. Beckenbodenebene) glei-

Fig. 12.

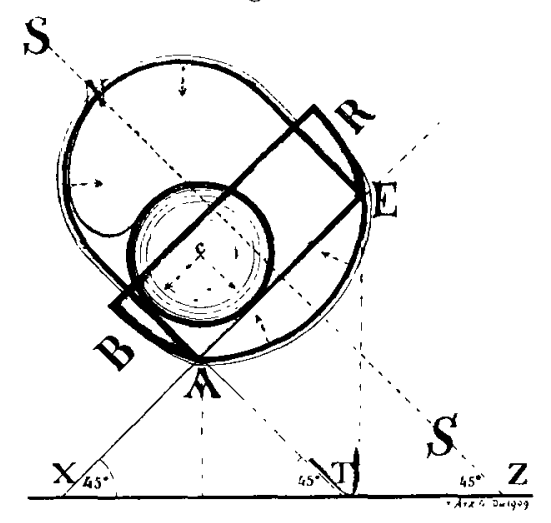

Blasenkugel $C$, Beckenring $B R$ und elastische Rumpfblase $A E X$ in physikalischer Relation bei der Elevation der Seelenachse $S S$ ron $45^{\circ}$. Die Blase schwebt im stabil-labilen Gleichgewicht. che Belastung statt in allen Fällen, wo diese beiden schiefen Ebenen nur gleiche Neigung zur Horizontalen besitzen (s. Fig. 13-15), d. h. einem gleichschenkligen $\gg$ Basisdreieck * aufsitzen. Der tiefstliegende Punkt $A$ des Beckenringes $B R$ 
sitzt also auf der Spitze eines gleichschenklig rechtwinkligen Dreiecks XTA (Fig. 12), dessen Hypotenusenwinkel je $45^{\circ}$ betragen. Da der Punkt $A$ dieser Figur im großen und ganzen dem Arcus (Angulus) pubis entspricht (Fig. 1 und 2), sind Fig. 13-15 genuigend orientiert.

Der freie Fall der Kugel $C$ wird, auf der Beckenbodenebene angelangt, durch zwei Momente gehemmt:

1) Zug des Aufhängebandes (Lig. med, vesicae) und

2) Gegendruck des Beckenbodens $A E$.

Nun ist der Innendruck der elastischen Rumpfblase ein stetig wechselnder. Er verändert sich nämlich nicht nur mit der wechseln-

Fig. 13.

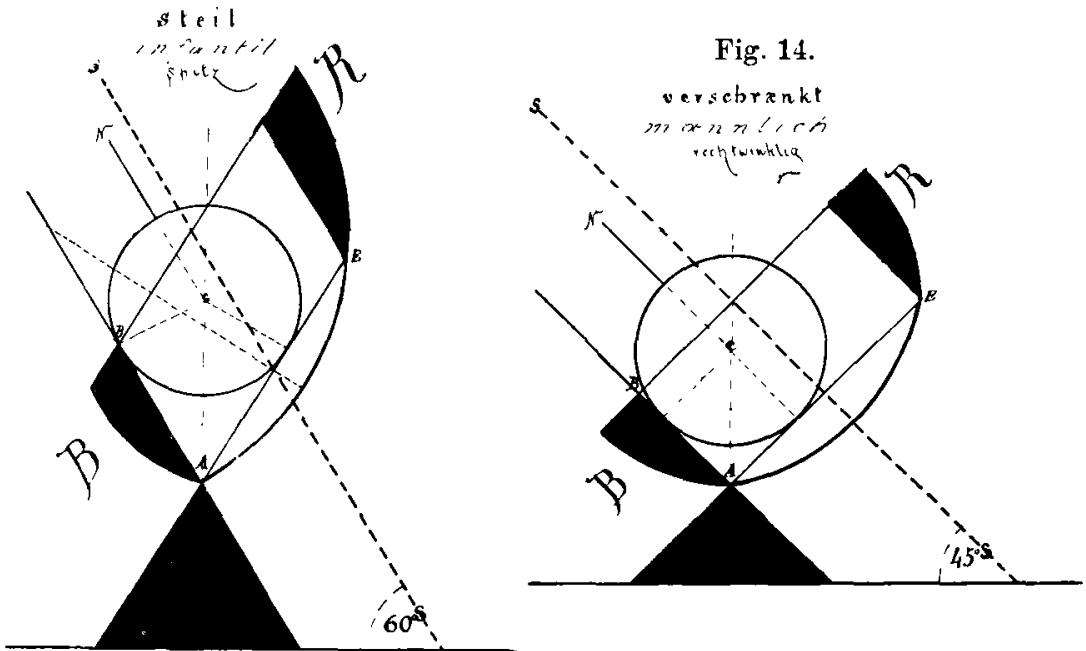

Fig. 13. Der steile Typus des infantilen Beckens.

Fig. 14. Der rechtwinklig verschrănkte Typus des männlichen Beckens.

den Füllung ihrer Eingeweide, sondern vor allem auch mit jedem Atemzug. Jede Druckschwankung macht sich aber an der Innenfläche der Rumpfwand geltend. Die einzelnen Partien dieser letzteren geben dem wachsenden Innendrucke nach, jedoch - wie bereits erörtert in verschiedenem Grade, je nach dem Elastizitätsvermögen der Wandung. Von allen Seiten wird der Druck reflektiert nach mechanischen Gesetzen. Dies geschieht also auch von seiten des Beckenbodens $A E$, wo in der Berechnung an Stelle des elastischen Kugelabschnittes $A E$ die Sehnenebene $A E$ tritt. Durch den elastischen Rückprall am Beckenboden wird anderseits die im Nabelpunkt $N^{\top}$ aufgehängte und fallende Blasenkugel $C$ auf die Beckenbodenebene zurückgeworfen und hier in stabil-labilem Gleichgewicht gehalten. 
Wie wir gesehen haben, lastet also die Kugel $C$ bei gleichschenkligem Basisdreieck mit ihrem Gewicht auf beiden Ebenen gleichmäßig. Der Druck auf jede dieser Ebenen ist $=$ dem eigenen Gewicht $\times \cos$ des Neigungswinkels. Dieser Satz gilt gleichmäßig für jede Größe des Basiswinkels. Welcher Vorzug kommt nun aber dem Winkel von $45^{\circ} \mathrm{zu}$ ? - Ein einziger Blick auf die Fig. 13, 14 und 15 mag zur Erklärung genïgen.

Beim Elevationswinkel von $45^{\circ}$ (Fig. 14) sind die Belastungsmomente für den Beckenring insofern am günstigsten, als sich bei rechtwinkliger Stellung der beiden Ebenen zueinander die Spannungsverhältnisse für den Ring und seinen Boden selber dabei am günstigsten gestalten. Dasselbe gilt auch für die äußeren Druckverhältnisse der Blase, da die Richtung des äußeren Druckes mit den Seiten des Belastungsparallelogramms zusammenfällt, parallel verläuft oder senkrecht zu denselben steht.

Näher auf die matheFig. 15.

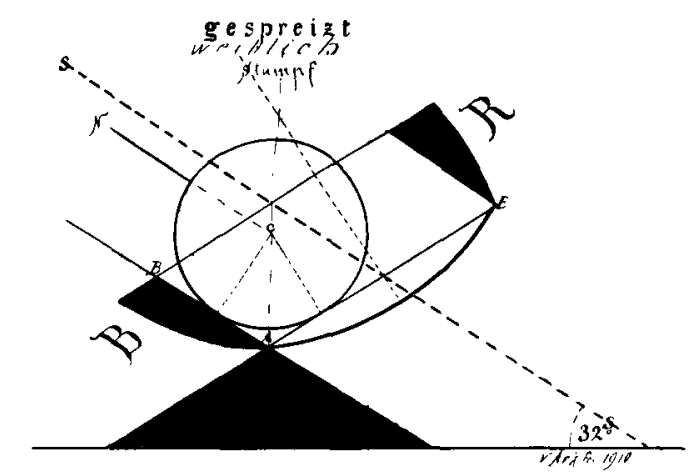

Der gespreizte Typus des weiblichen Beckens. matischen Berechnungen

dieser hypothetischen Grundbegriffe einzutreten, hätte vorderhand keinen Zweck. Es gentigt, darauf hingewiesen zu haben, daß in Wirklichkeit alle drei in Fig. 13-15 dargestellten Typen des Basisdreiecks mechanistiseh zur Anwendung kommen, und zwar:

das steile Basisdreieck beim juvenilen,

das rechtwinklig verschränkte Basisdreieck beim ausgewachsenen männlichen,

das gespreizte Basisdreieck beim ausgewachsenen weiblichen Becken.

Zur Vereinfachung der Denk- und Ausdrucksweise dürfen wir uns nach dieser erläuternden Auseinandersetzung nun auch des kürzeren und wissenschaftlich exakten Ausdrucks » steile «, »rechtwinklige * und gespreizte "Beckenform bedienen, müssen uns dabei aber ins Gedächtnis rufen, daß die Verbältnisse bei allen drei Beckenformen wohl rein im Prinzip, doch z. T. modifiziert, vor uns liegen. 
Im steilen Beckentypus des jugendlichen Individuums (Fig. 13) steht die Seelenachse $S S$ schief zur Beckenbodenebene $A E$, so daß das Perpendikel der letzteren mit ihr einen Winkel bildet; die Blasenkugel $C$ steht noch sehr hoch. Der tiefste Punkt $A$ des Beckenringes $B R$, der am menschlichen Becken dem Angulus pubis entspricht, ist also sowohl von der Peripherie des Kreises wie von den Endpunkten der Belastungsradien desselben weit entfernt. Die Bewegungsfähigkeit der Kugel ist in der gegebenen Lage eine beschränkte, indem sie durch die Kontraktion des Beckenbodens nur gegen den Beckenring und die vordere Bauchwand angedrickt wird. Statisch ist dieser Typus der ungüstigste von allen dreien, indem die Grundfläche seines Basisdreiecks die schmalste ist.

Der rechtwinklig verschränkte Typus (Fig. 14) vereinigt die Vorteile der Statik und Mechanik in sich. Seelenachse und Perpendikel des Beckenbodens verlaufen unter sich parallel oder fallen in der Richtung gänzlich zusammen. Die fallende Kugel belastet Beckenring und Beckenbodenebene gleichmäßig und gegen Einwirkung äußerer Kräfte, von welcher Seite sie auch kommen mögen, bietet statisch und mechanisch die rechtwinklige Verschränkung des Beckenringes und des Basisdreiecks den besten Widerstand. Wir haben diesen rechtwinklig verschränkten Typus dem ausgewachsenen männlichen Becken zugesprochen; dies mitssen wir jedoch cum grano salis verstehen.

Den oberen Winkel des Basisdreiecks, den wir als $\diamond$ Öffnungswinkel « bezeichnen und der bei diesem Typus genan $90^{\circ}$ betragen mïßte, fanden wir an unserm männlichen Becken bereits mebr gespannt. Er beträgt $105^{\circ}$, so daß die Basiswinkel dieses gleichschenkligen Dreiecks $\frac{180-105^{\circ}}{2}=37,5^{\circ}$ betragen, anstatt 45 . Trotzdem ist das männliche Becken nach allen Seiten hin rechtwinklig verschränkt, wie aus folgenden Tatsachen hervorgeht:

1) Vom Angulus pubis $A$, an der Spitze dieses Basisdreiecks, geht nach vorn die Verlängerung der einen Dreiecksseite zum Punkte $C$ (Tubercula pubis) an der Vertikalebene und bildet die vordere Symphysenfläche; nach hinten oben zielt die andre Dreiecksseite nach dem untersten Krenzbeinpunkte $E$. Der Öffnungswinkel zwischen diesen beiden Schenkeln ist nun entsprechend seinem entsprechenden Scheitelwinkel $105^{\circ}$, also gegenüber dem rechtwinkligen Basisdreieck um $15^{\circ}$ zu groß. Genau diese Differenz aber kommt nun der Dicke der Symphyse zugute, so daß die hintere Symphysen- 
fläche mit der Kreuzbeinebene $A E$ genau den rechten Winkel bildet. Das ist die eine rechtwinklige Verschränkung am männlichen Becken.

2) Ein zweites rechtwinkliges Achsenkreuz hat seinen Kreuzungspunkt im Scheitelpunkte $A$; es ist wieder um $15^{\circ}$ nach hinten gedreht und findet seine Stuitzpunkte im Nullpunkt $X$ des Koordinatensystems, an der Spitze des Steißbeins $D$ und an der Spina oss. ilei $(G)$.

3) Und drittens fällt die Vertikale vom Promontorium ebenfalls durch den Punkt $A$ und steht senkrecht zu der Horizontalen, die einerseits vorn die obere Grenze der Symphyse bildet und hinten durch die Steißbeinspitze geht.

Sind nicht diese Tatsachen allein schon überwältigend? Und doch sind sie nur von mehr sekundärer Bedeutung in der Statik des männlichen Beckens. Ich habe bereits vor Jahresfrist in Straßburg und Budapest darauf hingewiesen, daß die mathematische Grundform des männlichen Beckens die Kugel ist, mit drei senkrecht a ufeinander stehenden Achsen, und daß darin die Hauptstärke des männlichen Beckens liegt. Es wïrde hier zu Fig. 16.

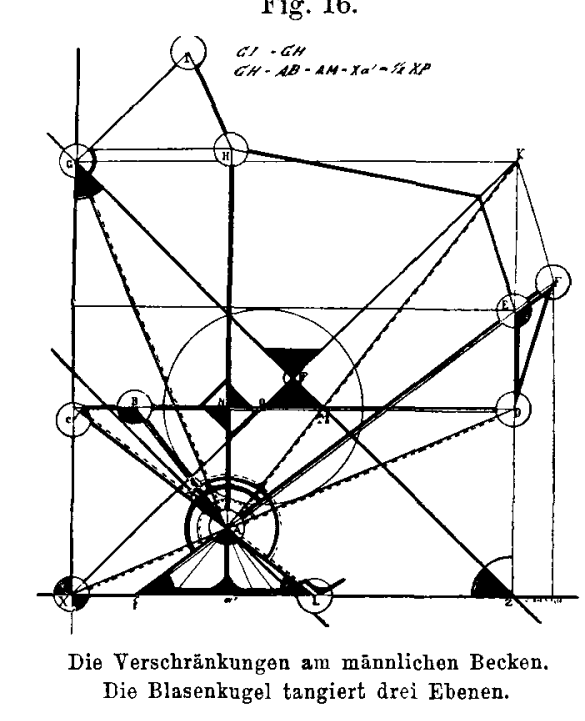
weit fuhhren, darauf einzugehen.

Die gespreizte Beckenform (Fig. 15) endlich finden wir normalerweise beim ausgewachsenen Weibe. Da die beiden Dreiecksschenkel und ihre Verlängerungen zur Horizontalen noch mehr geneigt sind, erhält die Kugel hier eine noch größere Beweglichkeit und Bewegungsfreiheit. Die mehr horizontale Lage des Beckenbodens gibt nämlich dem Perpendikel eine vertikalere Richtung, so daß die Kugel nun infolge der Kontraktion des elastischen Bodens nicht mehr wie im steilen Typus gegen die vordere Wand angedriuckt, sondern mehr vertikal nach oben geschleudert wird. - Am weiblichen Becken stehen nirgends mehr drei Achsen senkrecht aufeinander; wir finden daher nirgends mehr zwei Hauptebenen in senkrechter Lage 
zueinander stehen. Die mathematische Grundform des weiblichen Beckens ist die gesprengte Kugel, wobei die beiden Hemisphären in der Ebene der Seelenachse gesprengt werden und vertikal auseinander treten.

Das Wenige über die typischen Beckenformen mag andeutungsweise genügen und wir kehren wieder zum Mechanismus des Beckenbodens zurück.

Bisher haben wir tuberall mechanisch richtig an Stelle des Beckenbodenbogens seine Sehne gesetzt, in der Voraussetzung, daß die Kontraktionsfähigkeit, oder besser gesagt die Elastizität des Bogens, überall ungefähr die gleiche sei. Wir dürfen das auch für nnsre Zwecke ohne weiteres annehmen. Der Beckenring aber wurde in unsern Typen vorderhand als ein kurzes starres Cylinderrohr angesehen und hierin müssen wir nun unsre Ansicht gründlich ändern. Infolge der Elastizität und Dehnungsfähigkeit seiner Gewebe gibt der knöcherne Beckenring dem elastischen Beckenboden keine gänzlich unbeweglichen Fixpunkte ab. Stärkeren Spannungen in der Rumpfblase gegeniiber ist anch das Lumen des Beckenringes selber nicht widerstandsfähig genug. Ist ja doch, wie wir bereits einleitend bemerkt haben, der Beckenring nichts andres als ein Teil der Rumpfwandung. Diesen Satz müssen wir uns immer und immer wieder ins Gedächtnis zurïckrufen. Das Substrat des knöchernen Skelets aber ist der Knorpel und erst nach erfolgter Gleichgewichtslage werden die Kalksalze in denselben eingelagert. Daher paßt sich die Beckenform in allen Details den herrschenden Spannungsfaktoren an und aus der Form der Knochen können wir logischerweise auf die statischen und mechanischen Momente schließen, welche diese Knochenformen geschaffen haben.

Beim männlichen Becken nun ist der Mechanismus des Beckenbodens ein relativ einfacher. Der elastische Beckenboden spannt sich im Kreisbogen vom Angulus pubis zum Steißbein. Eine genaue seitliche Betrachtung des männlichen Beckens aber zeigt, daß dieser Kreisbogen viel weiter geht und auch vorn die Kontur der Schambeinfuge sowie die hintere Kontur des Kreuzbeins in sich faßt und zwar letztere bis zum oberen Ende des Hiatus canalis sacralis hinauf. Sonderbar ist, daß diese in die Augen springende Tatsache bisher in der Literatur unerwähnt geblieben ist! Die Steißbeinspitze $D$ kommt direkt unter den tiefsten Kreuzbeinpunkt $E$ zu liegen (Fig. 17). Die Folge davon ist die, daß das Steißbein in seiner Symphysenverbindung $E F$ mit rlem Kreuzbein am männlichen Becken ziemlich fest verbunden bleibt. 
Immerhin ist eine geringe Beweglichkeit des Steißbeins in toto, wie auch $\mathrm{zwischen}$ seinen einzelnen Knöchelchen, vorhanden; doch ist sie viel geringer, als dies am weiblichen Becken der Fall ist. Das Steißbein gilt daher beim Manne mechanisch als Teil des knöchernen Beckens und als Stitzpunkt für den elastischen Beckenboden $A D$ (Fig. 17). Der elastische Beckenboden bildet daher beim Manne eine einfache Federbrucke mit teilweise drehbaren Widerlagern.

Ganz anders gestaltet sich der Mechanismus beim Weibe, nicht etwa sowohl wegen der Verschiedenheit in der anatomischen Anord-

Fig. 17.

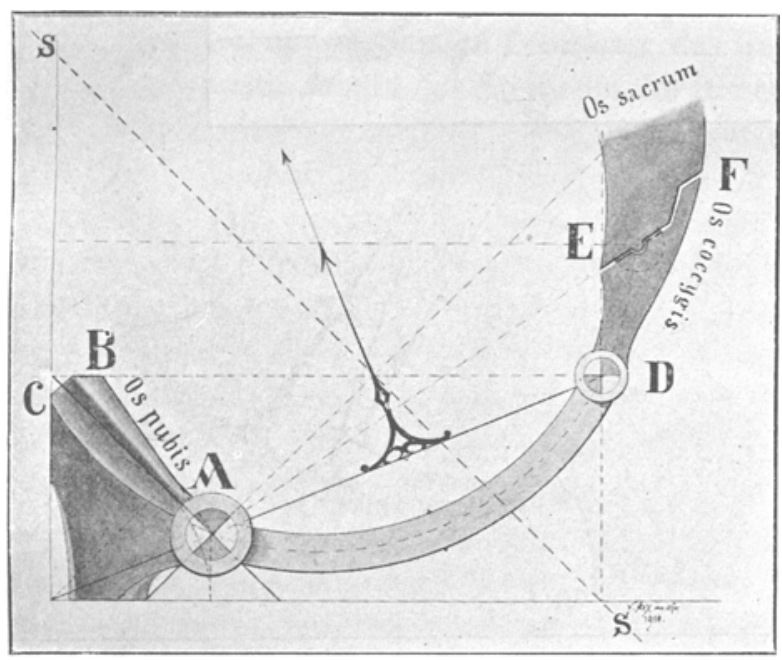

Mechanik des männlichen Beckenbodens als einfache elastische Feder dargestellt. Hängepunkte teilweise drehbar.

nung des Beckenbodens, - nein, der Unterschied im Mechanismus liegt tiefer begründet, in der viel stärkeren Spannung, welche in der Pubertätszeit des Weibes das juvenile Becken zum weiblichen Typus umzugestalten vermag. Über den prinzipiellen Unterschied der männlichen und weiblichen Beckenform finden wir in den Lehrbüchern der Anatomie und in denjenigen der Geburtshilfe nur sehr weniges und das wenige, das sich hierauf bezieht, trifft meist den Nagel nicht auf den Kopf.

Wie sich graphostatisch leicht nachweisen läßt, ist es die elastische Spannkraft des Uterus, welche die stärkere Ausweitung des Beckens in der Pubertätszeit bewirkt. 
Aber auch die änßeren Genitalien, vor allem die Schwellkörper, nehmen an der Formgestaltung des weiblichen Beckens regen Anteil; sie erweitern den Angulus zum Arcus pubis. Während so das Kreuzbein in gleichmäßigerer Wölbung nach hinten tritt, wird das Steißbein nach vorn gezogen (vgl. Fig. 1 und 2) und dadurch nicht nur in toto, sondern auch in sich selber bedeutend beweglicher gemacht.

Auf einen Punkt von wesentlicher mechanischer Bedeutung müssen wir hier noch zu sprechen kommen, auf die Bedentung der Cornua coccygea des ersten Steißbeinwirbels, die noch als deutliche Überreste der oberen Processus obliqui vorhanden sind. Mechanisch prä-

Fig. 18.

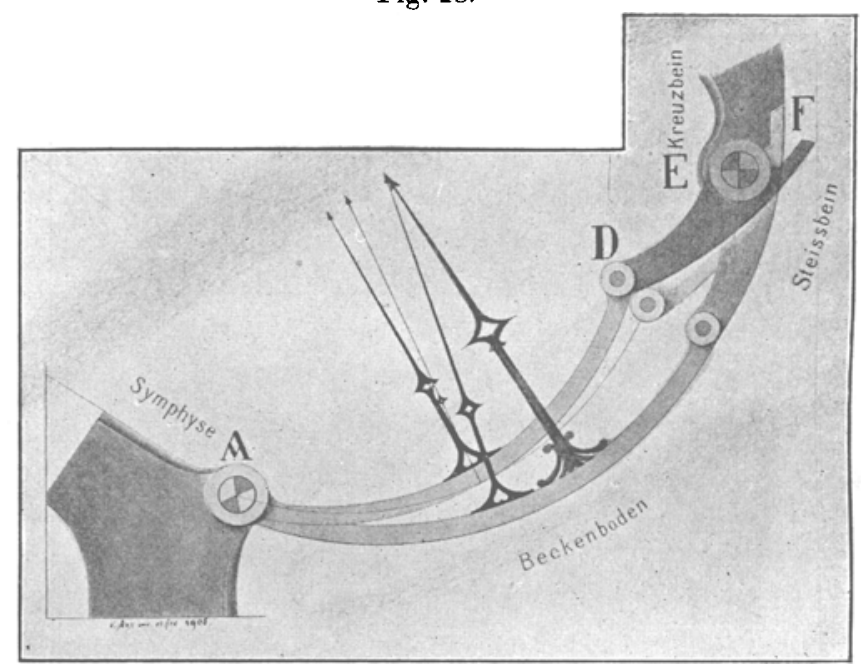

Mechanik des weiblichen Beckenbodens als gelenkig verbundene Doppelfeder dargestellt.

sentieren sie sich vorzüglich als Sperrvorrichtung oder Retentaculum gegen einen Überdruck auf den Beckenboden von innen her.

Die veränderte Stellung des Steißbeins am weiblichen Becken, sowie seine vermehrte Beweglichkeit und Krümmungsfähigkeit geben nun diesem Organ eine ganz andre mechanistische Bedeutung, als sie ihm beim männlichen Beckentypus noch zukommt. Das Steißbein ist nämlich selbst als eine Feder zu betrachten; diese Eigenschaft kommt jedoch erst bei stärkerem Innendruck der Bauchhöhle zur Geltung. Bei normalem Druck gestalten sich die Verhältnisse analog dem männlichen Becken, indem die Steißbeinspitze als Ansatzpunkt für den elastischen Beckenboden zu betrachten ist. Dabei denken wir uns die Articulationen zwischen den einzelnen Steißbeinknöchelchen gleichwie beim 
Manne ruhiggestellt. Bei wachsendem Innendruck der Bauch-Rumpfhöhle aber treten auch diese Gelenke in Funktion, und zwar um so mehr, je mehr sie sich nach unten zu dem elastischen Beckenboden nähern. Mit der Dehnung des letzteren tritt also auch die Steißbeinspitze nach außen. Diese Streckung des Os coccygeum kann erfolgen bis zu dem Punkt, wo seine äußere Krtimmung die Fortsetzung bildet des maximal gespannten elastischen Beckenbodens. In diesem Moment tritt das Retentaculum der Cornua coccygea in Funktion und verhindert jede weitere Dehnung des Beckenbodens. Die Mechanik des weiblichen Beckenbodens ist daher diejenige einer elastischen Doppelfeder mit mittlerem Charnier und drehbaren Auflagern (Fig. 18).

Wir haben eben von der maximalen Spannung des Beckenbodens gesprochen, welche eintritt, sobald das Steißbein die Bogenkrummung des elastischen Bogens eingenommen hat. Dieser Satz muß mit Vorbehalt ausgesprochen werden. Bei maximalster Spannung der Bauchhöhle, wie sie beim Weibe sowohl physiologischer wie pathologischer Weise (Schwangerschaft, Tumorenbildung und Ascites) vorkommen, bemerkt man äußerlich eine Schwellung und Vorwölbung der vorderen Beckenbodengegend, und nur dieser. Die äußeren Genitalien werden nicht nur succulent, sondern auch vorgetrieben. Es ist daher auch die Vorbereitung der äußeren Genitalien zur Geburt durchaus ein mechanischer Vorgang. Er beruht auf der Überdehnung der vorderen Hälfte des Beckenbodens, welche beim Weibe schwächer ist infolge der Anlage des Introitus vaginae. Daß dasselbe Phänomen auch bei pathologischer Spannung beim Weibe, und nur bei diesem auftritt, bestätigt die Richtigkeit dieser Annahme.

Im engsten Zusammenhang mit dem Mechanismus des Beckenbodens steht Statik und Mechanik der Blasenkugel. Was diese beim männlichen Individuum anbetrifft, haben wir hier dem oben Gesagten nicht mehr viel beizufügen. Die Verhältnisse sind durchaus einfache und klare; auch entsprechen sie nach unsern Untersuchungen durchaus den topographisch-anatomischen Angaben, wie sie s. Z. P. Trllaux für die Lage der Blase angegeben hat.

Schwieriger gestalten sich die Verhältnisse beim Weibe, und da muß man gleich von vornherein verschiedenen landläufigen Auffassungen entgegentreten, wie sie heute immer noch an den meisten Kliniken gelehrt werden. Wir stellen diesbezüglich folgende Sätze auf:

1) Wird unter normalen Verhältnissen durch maximale Füllung der Blase der Uterus niemals nach hinten an das Rectum angedrängt, 
aus dem einfachen Grunde nicht, weil die seitliche Spannung der Lig. rotunda den Uterus stets nach vorn zieht und so eine maximale Dehnung im unteren Blasenteil nicht zuläßt (s. Archiv f. Anat. 1898 und Korresp. f. Schw. Ä. 1896). Der Douglassche Raum fullt sich alsdann mit den Darmschlingen, die aus der Bauchhöhle verdrängt werden.

2) Der Uterus ist mechanisch nicht als ein $\mathrm{zweiarmiger,} \mathrm{sondern}$ eher als ein einarmiger Hebel anzusehen, dessen wenn auch nicht ganz festgestellter Drehpunkt oben am Ansatz der Lig. rotunda zu suchen ist.

3) Was beim Prolaps zuerst vorfällt, ist nicht die vordere Scheidenwand oder der Uterus. Die treibende Kraft, das Agens movens, ist die Schwerkraft der toten Masse, des beweglichen flussigen Blaseninhaltes, sobald die Spannkraft des Beckenbodens infolge von Dammriß usw. verloren geht.

Mechanisch wie genetisch bilden Blase und Uterus ein unzertrennbares Ganzes. Nur zwei Punte verbinden das Gebärorgan embryologisch mit seiner Nachbarschaft: einerseits die Lig. rotunda mit der vorderen Bauchwand und anderseits die feste Vereinigung des Collum mit der hinteren Blasenwand; denn was das Lig. latum anbetrifft, sowie die andern verschiedenen Plicae serosae, so sind sie durchaus nebensächliche Gebilde ohne jede mechanische Bedeutung.

Der Drehpunkt des Uterus kommt nun auf das Perpendikel aes Beckenbodens zu liegen. Der Uterus würde also, wenn er nicht in einer geschlossenen Bauchhöhle läge und vom Blasenfundus dirigiert nach hinten gedrängt würde, genau in der Richtung dieses Perpendikels hängen. Das ist tatsächlich auch der Fall im Anfang, wo er seiner Elastizität aus diesem oder jenem Grunde verloren geht. Die Abknickung des normalen Uterus aber ist die alleinige Folge der Rückwirkung des Innendruckes der Bauchhöhle von der hinteren Beckenwand.

Diese Andeutungen mögen genügen. Sie sind durch graphostatische Konstruktion leicht zu erbärten.

Es ist ja nichts mehr als eine logische Schlußfolgerung, wenn wir aus der äußeren » funktionellen Gestalt « (Roux) der Knochen umgekehrt auf die statischen und mechanischen Momente schließen können, welche diese Knochenform geschaffen haben. Freilich werden dabei die bisherigen Anschauungen der Anatomen und Kliniker bis in ihre Grundfesten erschittert und wir gelangen zu ganz andern 
Vorstellungen über unser physisches Werden und Sein. Aber diese neuen Vorstellungen vereinfachen in nie geahnter Weise das physiologisch-anatomische Denken, indem sie uns auf den sicheren Boden mathematischer Deduktionen führen.

Unsre Untersuchungen greifen jedoch hintiber in zahlreiche andre Gebiete der Forschung und müssen auf dem Feld der Entwicklungsgeschichte und Entwicklungsmechanik, der vergleichenden Anatomie und Anthropogenese weitergefuhrt werden. Die Erfolge, welche die darstellende Geometrie in der Erkenntnis des menschlichen Beckens gebracht, die Koinzidenz jeder anatomischen Kontur an demselben mit der mathematisch konstruierten Linie, das Auffinden endlich des einfachen Naturgesetzes elementarster Spannkräfte, sie werden alle der mechanistischen Weltanschaung eine Stlitze sein. 